\title{
Linear stability of thermocapillary convection in cylindrical liquid bridges under axial magnetic fields
}

\author{
By M. PRANGE†, M. WANSCHURA, \\ H. C. KUHLMANN AND H. J. RATH \\ Center of Applied Space Technology and Microgravity, ZARM - University of Bremen, \\ Am Fallturm, D-28359 Bremen, Germany
}

(Received 15 December 1997 and in revised form 10 April 1999)

The stability of axisymmetric steady thermocapillary convection of electrically conducting fluids in half-zones under the influence of a static axial magnetic field is investigated numerically by linear stability theory. In addition, the energy transfer between the basic state and a disturbance is considered in order to elucidate the mechanics of the most unstable mode. Axial magnetic fields cause a concentration of the thermocapillary flow near the free surface of the liquid bridge. For the low Prandtl number fluids considered, the most dangerous disturbance is a non-axisymmetric steady mode. It is found that axial magnetic fields act to stabilize the basic state. The stabilizing effect increases with the Prandtl number and decreases with the zone height, the heat transfer rate at the free surface and buoyancy when the heating is from below. The magnetic field also influences the azimuthal symmetry of the most unstable mode.

\section{Introduction}

\subsection{Motivation}

Flows driven by thermally induced surface tension gradients occur in many different physical systems and are of paramount importance for certain crystal growth processes like the floating-zone technique. In the floating-zone configuration a polycrystalline feed rod is passed through a ring heater, producing a melt zone that is held by surface tension forces. Thermocapillary-driven flow in the melt strongly influences the distribution of dopants and impurities in the growing crystal (Eyer \& Leiste 1985; Cröll, Müller-Sebert \& Nitsche 1989; Cröll et al. 1991).

Magnetic fields can be used to control the melt convection during the float-zoning of semiconductor crystals. The motion of the electrically conducting melt under a magnetic field induces electric currents. Lorentz forces, resulting from the interaction between the electric currents and the magnetic field, affect the flow. In particular, convective instabilities can be suppressed to a considerable degree (e.g. Chandrasekhar 1961). Experiments of Herrmann et al. (1992) and Cröll, Dold \& Benz (1994) showed that steady magnetic fields can improve the micro-homogeneity of float-zone grown semiconductor single crystals by suppressing unsteady thermocapillary convection. A

$\dagger$ Present address: Alfred-Wegener-Institute for Polar and Marine Research, Am Handelshafen 12, D-27570 Bremerhaven, Germany. 
basic understanding of thermocapillary flows, their instabilities and their stabilization by magnetic fields is required in order to optimize crystal growth processes.

\subsection{Previous studies}

Owing to the complexity of real floating zones (deformability of the free surface, deformable phase changing surfaces, rotating crystal rods, external flows, intricate thermal boundary conditions, etc.) most theoretical studies consider highly idealized models. The most common model is that of a cylindrical liquid bridge between two rigid planar disks. Keeping the supporting disks at different temperatures induces an axial surface tension gradient and thus thermocapillary convection. This configuration is known as the half-zone model and has been used in the numerical studies of Rupp, Müller \& Neumann (1989), Kuhlmann \& Rath (1993), Levenstam \& Amberg (1995), Wanschura et al. (1995) and others. As long as the imposed temperature difference is sufficiently small the flow field consists of a steady axisymmetric vortex. If the temperature difference exceeds a critical value, the basic flow becomes unstable.

Rupp et al. (1989) and Levenstam \& Amberg (1995) carried out three-dimensional time-dependent simulations and found that the first instability of the axisymmetric basic state in low Prandtl number fluids $(\operatorname{Pr} \ll 1)$, like liquid metals and semiconductor melts, is stationary and non-axisymmetric (i.e. three-dimensional). Time-dependent flow occurred only after a second instability in the simulations. Kuhlmann \& Rath (1993) and Wanschura et al. (1995) investigated the stability of the axisymmetric basic state by means of linear stability theory and studied wide ranges of parameters. Their results confirm the stationary and non-axisymmetric first instability found in the simulations of Rupp et al. (1989) and Levenstam \& Amberg (1995) for small Prandtl numbers. Wanschura et al. (1995) demonstrated the strong dependence of the three-dimensional flow's azimuthal symmetry on the aspect ratio (i.e. height/radius) of the half-zone. The study pointed out that the azimuthal wavenumber increases almost linearly with the inverse aspect ratio. By means of an energy analysis Wanschura et al. (1995) examined the physical instability mechanism and found that the low Prandtl number instability is due to the large strain rate present in the basic axial shear flow. For large Prandtl numbers (i.e. $\mathrm{Pr}>0.5$ ) the axisymmetric basic flow becomes linearly unstable to a pair of hydrothermal waves propagating nearly azimuthally which result from a synchronous coupling of conductive and convective heat transports. Kuhlmann \& Rath (1993) studied the effect of heat loss through the free surface and showed a destabilizing influence on the axisymmetric flow in low Prandtl number fluids. Moreover, the inclusion of weak buoyancy forces in their model acted to stabilize the basic state when the liquid was heated from above and to destabilize when the heating was from below.

Apart from the work of Baumgartl et al. (1990) who demonstrated the damping effect of axial magnetic fields on the unsteady convection in a full-zone configuration by means of a time-dependent three-dimensional simulation, there have been no numerical studies on the influence of magnetic fields on the stability of thermocapillary flow in liquid bridges. Baumgartl et al. (1990) considered the thermocapillary flow driven by an imposed parabolic heat flux profile at the free surface. In their calculation induced electric fields were neglected. As we shall see in $\S 4.2$, however, this approximation is not justified. The numerical results for magnetic field effects on thermocapillary flows in Czochralski configurations (see e.g. Khine \& Walker 1994 and references therein) or infinite plane layers (e.g. Priede, Thess \& Gerbeth 1994; Priede \& Gerbeth 1995; see also the review in Wilson 1994) cannot be applied to the floating-zone problem owing to the different geometry. 


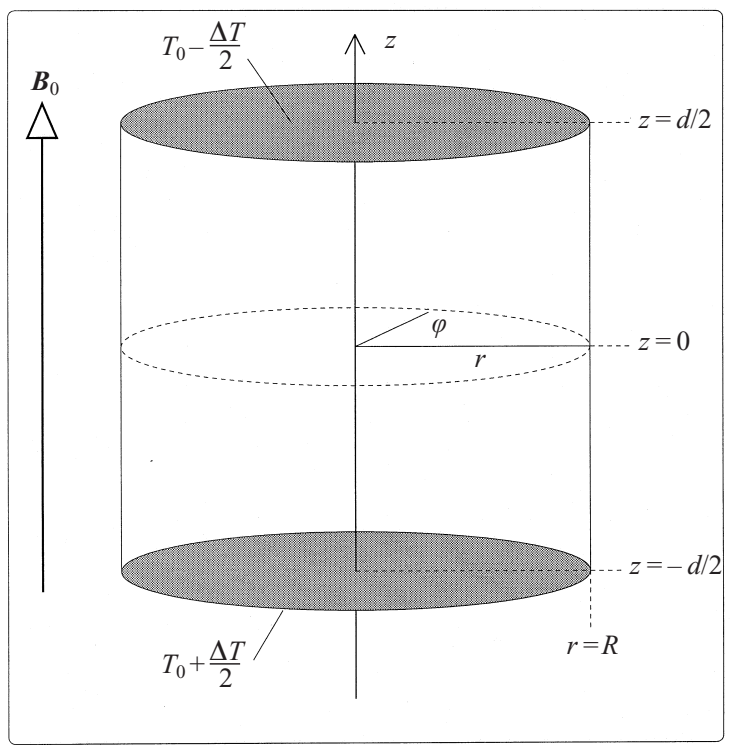

FIGURE 1. Geometry of the half-zone. The liquid bridge is subject to a static axial magnetic field $\boldsymbol{B}_{0}$. Cylindrical coordinates are used.

\subsection{This study}

In the present work we examine the influence of a steady axial magnetic field on the stability of axisymmetric thermocapillary convection in half-zones by means of linear stability theory, i.e. we extend the problem studied by Kuhlmann \& Rath (1993) and Wanschura et al. (1995) to include magnetohydrodynamic effects for low Prandtl number fluids. Several parameter variations are performed to obtain deeper insights into the nature of the convective instability and its suppression by magnetic fields. Systematically, we study the influence of geometry (aspect ratio), Prandtl number, heat exchange with the ambient medium, and buoyancy forces. To this end we adopt the numerical methods of Wanschura et al. (1995) where the model equations are discretized by a mixed Chebychev-finite difference scheme. The linear stability calculations are accompanied by energy analyses which serve a double purpose. They provide a check of the linear stability analysis and reveal the physical mechanisms which stabilize or destabilize the basic flow.

\section{Statement of the problem}

We consider a liquid bridge between two parallel concentric rigid disks of radius $R$ which are separated by a distance $d$ (figure 1). A temperature gradient is imposed on the fluid volume by keeping the disks at different temperatures $T(z=-d / 2)=$ $T_{0}+\Delta T / 2$ and $T(z=d / 2)=T_{0}-\Delta T / 2$, where $T_{0}$ denotes the mean temperature and $\Delta T$ the temperature difference. Heating the liquid bridge from below corresponds to a positive sign of $\Delta T$. In the limit of large mean surface tension $\sigma_{0}$ static and dynamic deformations of the free surface can be neglected and, for an appropriate volume, the fluid takes a cylindrical shape. The aspect ratio is defined by

$$
\Gamma=\frac{d}{R} \text {. }
$$


Since surface tension decreases with increasing temperature a thermocapillary upward flow (provided that $\Delta T$ is positive) is induced at the free surface and a steady axisymmetric vortex arises. As long as the imposed temperature difference does not exceed a critical value the toroidal basic flow remains stable. In the present study the effect of a static uniform axial magnetic field on the stability of the basic flow is investigated.

For small magnetic Reynolds numbers

$$
R e_{\mathrm{m}}=\mu_{0} \sigma_{\mathrm{el}} U L \ll 1
$$

( $\mu_{0}$ magnetic field constant, $\sigma_{\mathrm{el}}$ electrical conductivity of the fluid, $U$ characteristic velocity, $L$ length scale) the magnetic field induced by electric currents in the fluid is negligible (see e.g. Moreau 1990). In typical melt zones of semiconductor crystal growth $R e_{\mathrm{m}}$ does not exceed $10^{-3}$. Thus, we can assume that the magnetic induction $\boldsymbol{B}$ is that of the applied steady field $\boldsymbol{B}_{0}$. Consequently, induced electric fields can be expressed by the negative gradient of an electrical potential $\phi$. Using the scalings $d$, $v / d, \rho_{0} v^{2} / d^{2}, \Delta T, d^{2} / v$ and $v B_{0}$ for length, velocity, pressure, temperature, time and electric potential, where $v$ and $\rho_{0}$ denote the kinematic viscosity and the mean density, respectively, we obtain the following dimensionless governing equations in Boussinesq approximation and (dimensionless) cylindrical coordinates $(r, \varphi, z)$ :

$$
\begin{gathered}
\nabla \cdot \boldsymbol{u}=0, \\
\partial_{t} \boldsymbol{u}+(\boldsymbol{u} \cdot \nabla) \boldsymbol{u}=-\nabla p+\Delta \boldsymbol{u}+G r \theta \boldsymbol{e}_{z}+H a^{2}\left(\boldsymbol{u} \times \boldsymbol{e}_{z} \times \boldsymbol{e}_{z}-\nabla \phi \times \boldsymbol{e}_{z}\right), \\
\partial_{t} \theta+(\boldsymbol{u} \cdot \nabla) \theta=w+\frac{1}{P r} \Delta \theta, \\
\Delta \phi=\nabla \cdot\left(\boldsymbol{u} \times \boldsymbol{e}_{z}\right),
\end{gathered}
$$

where $p$ is the dimensionless pressure. The components of the velocity field $\boldsymbol{u}$ are $(u, v, w)$ (radial, azimuthal, axial) and $\theta$ describes the deviation from the conducting linear temperature profile

$$
T=T_{0}+\Delta T(\theta-z) .
$$

The dimensionless parameters arising are known as the Grashof, Hartmann and Prandtl numbers, defined by

$$
\begin{gathered}
G r=\frac{\beta g \Delta T d^{3}}{v^{2}}, \\
H a=\left(\frac{\sigma_{\mathrm{el}}}{\rho_{0} v}\right)^{1 / 2} d B_{0}, \\
P r=\frac{v}{\kappa},
\end{gathered}
$$

where $\beta, g$ and $\kappa$ denote the thermal expansion coefficient, the acceleration due to gravity, and the thermal diffusivity, respectively. The momentum equation (4) contains the Lorentz force term. Lorentz forces are proportional to the vector product of the induced (dimensionless) electric currents (Ohm's law)

$$
\boldsymbol{j}=\boldsymbol{u} \times \boldsymbol{e}_{z}-\nabla \phi
$$

and the applied magnetic field $\boldsymbol{B}_{0}=B_{0} \boldsymbol{e}_{z}$. The Poisson equation (6) for the electric potential is obtained by taking the divergence of (11) and considering the conservation of electric charge, i.e. $\nabla \cdot \boldsymbol{j}=0$. 
The boundary conditions at the rigid walls $\left(z=-\frac{1}{2}, \frac{1}{2}\right)$ read

$$
\boldsymbol{u}=\theta=\partial_{z} \phi=0,
$$

where we demand that no electric currents penetrate into the disks. The thermal condition at the free surface $(r=1 / \Gamma)$ is given by Newton's heat transfer law, assuming a linear axial profile for the ambient temperature:

$$
\left(\partial_{r}+B i\right) \theta=0,
$$

with the Biot number

$$
B i=\frac{h d}{\lambda},
$$

where $h$ and $\lambda$ denote the heat transfer coefficient and the thermal conductivity, respectively. The electrical boundary condition at $r=1 / \Gamma$ reads

$$
v-\partial_{r} \phi=0,
$$

since electric currents may not leave the fluid. The balance of tangential stresses at the free surface (see e.g. Kuhlmann \& Rath 1993) in conjunction with a linear approach for the temperature-dependence of the surface tension $\sigma$

$$
\sigma(T)=\sigma_{0}\left(T_{0}\right)-\gamma\left(T-T_{0}\right), \quad \gamma=-\frac{\partial \sigma}{\partial T}\left(T_{0}\right),
$$

yields the following thermocapillary boundary conditions:

$$
\begin{gathered}
\left(\partial_{r}-\Gamma\right) v+\operatorname{Re} \Gamma \partial_{\varphi} \theta=0, \\
\partial_{r} w+\operatorname{Re}\left(\partial_{z} \theta-1\right)=0 .
\end{gathered}
$$

The thermocapillary Reynolds number $R e$ is defined as

$$
R e=\frac{\gamma \Delta T d}{\rho_{0} v^{2}} .
$$

For given fluid and geometry $R e$ is simply proportional to the imposed temperature difference. The radial velocity vanishes at $r=1 / \Gamma$ since we do not allow for surface deformations.

Note that the thermocapillary Reynolds number (19) corresponds to the conventional Reynolds number $U d / v$ only for weak thermocapillary flow $(\operatorname{Re} \rightarrow 0, \operatorname{Pr} \rightarrow 0)$ without magnetic field for which the appropriate scaling of velocity is $U=\gamma \Delta T /\left(\rho_{0} v\right)$ (e.g. Kuhlmann 1995). The Marangoni number, also frequently used to characterize thermocapillary flows, is defined as $M a=R e P r$.

\section{Numerical methods}

\subsection{Basic state}

The axisymmetric steady basic state is characterized by $\partial_{t}=\partial_{\varphi}=v_{0}=0$ (the subscript 0 indicates basic-state quantities). To calculate the velocity field we use a stream function-vorticity formulation, where stream function $\psi_{0}$ and vorticity $\Omega_{0}$ are defined as

$$
\begin{gathered}
u_{0}=\partial_{z} \psi_{0}, \\
w_{0}=-\mathrm{D} \psi_{0}, \\
\Omega_{0}=-\partial_{r} w_{0}+\partial_{z} u_{0}
\end{gathered}
$$


with $\mathrm{D}:=\partial_{r}+1 / r$. The Stokes stream function is given by $\Psi=r \psi_{0}$. Taking the curl of (4) (and expressing the velocity components through stream function and vorticity) yields the bulk equations

$$
\begin{gathered}
\left(\partial_{r} \mathrm{D}+\partial_{z}^{2}\right) \psi_{0}-\Omega_{0}=0 \\
\left(\partial_{r} \mathrm{D}+\partial_{z}^{2}-\partial_{z} \psi_{0} \partial_{r}+\mathrm{D} \psi_{0} \partial_{z}+\frac{1}{r} \partial_{z} \psi_{0}\right) \Omega_{0}-G r \partial_{r} \theta_{0}-H a^{2} \partial_{z}^{2} \psi_{0}=0 \\
\left(\mathrm{D} \partial_{r}+\partial_{z}^{2}-\operatorname{Pr} \partial_{z} \psi_{0} \partial_{r}+\operatorname{Pr} \mathrm{D} \psi_{0} \partial_{z}\right) \theta_{0}-\operatorname{Pr} \mathrm{D} \psi_{0}=0 .
\end{gathered}
$$

For axisymmetric flow (6) and (15) reduce to $\Delta \phi_{0}=0$ and $\partial_{r} \phi_{0}=0$, respectively. The solution of the Laplace equation with Neumann boundary conditions (12) and (15) is trivial and reads

$$
\nabla \phi_{0}=0,
$$

meaning that no electric fields are induced. Thus, according to Ohm's law (11), electric currents are always perpendicular to the velocity and the magnetic field vectors, i.e. purely azimuthal. Consequently, Lorentz forces are directed radially and opposite to $u_{0}$, resulting in a flow retardation. Using the boundary conditions

$$
\psi_{0}=\partial_{z} \psi_{0}=\theta_{0}=0
$$

at $z=-\frac{1}{2}, \frac{1}{2}$,

$$
\psi_{0}=\Omega_{0}=\partial_{r} \theta_{0}=0
$$

at $r=0$, and

$$
\begin{gathered}
\psi_{0}=0, \\
-\Omega_{0}+\operatorname{Re}\left(\partial_{z} \theta_{0}-1\right)=0, \\
\left(\partial_{r}+B i\right) \theta_{0}=0
\end{gathered}
$$

at the free surface, we solve the basic-state problem with the numerical code of Wanschura et al. (1995) modified for the magnetic case as follows. The set of differential equations is discretized by means of a Chebyshev collocation method (Canuto et al. 1988) in the radial direction and a second-order finite-difference scheme in the axial direction. The resulting algebraic system is solved implicitly by Newton iteration (e.g. Schwarz 1993). The discretization grid consists of equidistant points in the axial direction and non-equidistant Gauss-Lobatto-points in the radial direction. Gauss-Lobatto-points are defined as

$$
x_{j}=\cos \left(\pi \frac{j}{M}\right), \quad j=0, \ldots, M,
$$

where $M$ denotes the number of radial grid points and $x=2 \Gamma r-1$. Due to the non-equidistant distribution of the radial grid points flow structures close to the free surface are efficiently resolved.

3.2. Linear stability analysis

Having calculated the axisymmetric basic state, its stability is investigated by a linear analysis. To obtain a sufficient condition for instability infinitesimal perturbations are 
superimposed onto the basic-state solution:

$$
(\boldsymbol{u}, p, \theta, \phi)=\left(\boldsymbol{u}_{0}, p_{0}, \theta_{0}, \phi_{0}\right)+\left(\boldsymbol{u}_{\mathrm{p}}, p_{\mathrm{p}}, \theta_{\mathrm{p}}, \phi_{\mathrm{p}}\right)
$$

(the subscript $p$ indicates perturbation fields). Substitution into (3)-(6) and linearization with respect to the perturbation quantities yields

$$
\begin{gathered}
\nabla \cdot \boldsymbol{u}_{\mathrm{p}}=0, \\
\partial_{t} \boldsymbol{u}_{\mathrm{p}}+\left(\boldsymbol{u}_{\mathrm{p}} \cdot \nabla\right) \boldsymbol{u}_{0}+\left(\boldsymbol{u}_{0} \cdot \nabla\right) \boldsymbol{u}_{\mathrm{p}}=-\nabla p_{\mathrm{p}}+\Delta \boldsymbol{u}_{\mathrm{p}}+G r \theta_{\mathrm{p}} \boldsymbol{e}_{z} \\
+H a^{2}\left(\boldsymbol{u}_{\mathrm{p}} \times \boldsymbol{e}_{z} \times \boldsymbol{e}_{z}-\nabla \phi_{\mathrm{p}} \times \boldsymbol{e}_{z}\right), \\
\partial_{t} \theta_{\mathrm{p}}+\left(\boldsymbol{u}_{\mathrm{p}} \cdot \nabla\right) \theta_{0}+\left(\boldsymbol{u}_{0} \cdot \nabla\right) \theta_{\mathrm{p}}=w_{\mathrm{p}}+\frac{1}{P r} \Delta \theta_{\mathrm{p}}, \\
\Delta \phi_{\mathrm{p}}=\nabla \cdot\left(\boldsymbol{u}_{\mathrm{p}} \times \boldsymbol{e}_{z}\right) .
\end{gathered}
$$

The general solution of this linear system can be expressed by a superposition of normal modes

$$
\mathscr{F}_{\mathrm{p}}(r, \varphi, z, t)=\mathrm{e}^{\tilde{\alpha} t} \mathrm{e}^{\mathrm{i} m \varphi} \mathscr{F}^{\prime}(r, z)+\text { c.c. },
$$

where $\mathscr{F}$ represents the field quantities and $m$ is an integer azimuthal wavenumber. Here and in the following primes denote $(r, z)$-dependent perturbation amplitudes. Generally $\tilde{\alpha}=\alpha+\mathrm{i} \omega$ is complex with growth rate $\alpha$ and oscillation frequency $\omega$. For a mode of neutral stability $\alpha=0$, while unstable/stable modes have positive/negative growth rates.

The further treatment of the stability problem requires the distinction between axisymmetric $(m=0)$ and non-axisymmetric $(m \geqslant 1)$ perturbations. For the axisymmetric case we apply the stream function-vorticity approach according to (20)-(22) similar to the basic-state calculation. As we shall see below, however, axisymmetric modes are not important for the instability. Therefore, we omit the detailed description of the axisymmetric stability problem (for the non-magnetic case the reader is refered to Wanschura et al. 1995) and consider only the three-dimensional case. We express the azimuthal velocity component $v^{\prime}$ in terms of $u^{\prime}$ and $w^{\prime}$ using the continuity equation

$$
v^{\prime}=\frac{\mathrm{i} r}{m}\left(\mathrm{D} u^{\prime}+\partial_{z} w^{\prime}\right) .
$$

Thus, the problem is reduced to five scalar quantities $\left(u^{\prime}, w^{\prime}, p^{\prime}, \theta^{\prime}, \phi^{\prime}\right)$. Inserting (38) and the normal mode approach (37) into (33)-(36), keeping the radial and the axial momentum equations, and taking the divergence of (34) we obtain the linear system of partial differential equations given in the Appendix. Using the same discretization scheme as for the basic-state calculation, we finally have a generalized eigenvalue problem

$$
\boldsymbol{A} \boldsymbol{x}=\tilde{\alpha} \boldsymbol{B} \boldsymbol{x},
$$

where $\boldsymbol{A}$ and $\boldsymbol{B}$ are the matrix representations of the set of linear volume equations and boundary conditions and $\boldsymbol{x}$ denotes the eigenvector of field quantities. The flow is linearly unstable if at least one eigenvalue exists which has a positive real part. Hence, the linear stability boundary is given by the set of parameters for which the largest real part of all eigenvalues vanishes. Inverse iteration (Press et al. 1989) is applied to (39) in order to find this mode for a given set of parameters $(B i, G r, H a, P r, \Gamma, m)$. The neutral Reynolds number $R e_{c}^{(m)}$ for a given azimuthal wavenumber $m$ is determined 
by

$$
\alpha\left(B i, G r, H a, \operatorname{Pr}, \Gamma, \operatorname{Re}_{c}^{(m)}, m\right)=0 .
$$

The critical Reynolds number is

$$
\operatorname{Re}_{c}(B i, G r, H a, P r, \Gamma)=\min _{m}\left\{R e_{c}^{(m)}(B i, G r, H a, P r, \Gamma)\right\} .
$$

The corresponding mode of disturbance is called the most dangerous, most unstable, or critical mode. Stability calculations for the present work were performed with wavenumbers $m$ ranging from 0 to 6 .

\subsection{Energy analysis}

Calculating the energy transfer between the basic state and the critical mode of disturbance provides a check (conservation of energy) of the linear stability analysis and may reveal the physical mechanisms which tend to stabilize or destabilize the basic flow. The rate of change of the kinetic energy $E_{\text {kin }}$ is obtained by multiplying (34) by $\boldsymbol{u}_{\mathrm{p}}$ and integrating over the entire cylindrical volume $V$. Taking into account the boundary conditions the Reynolds-Orr energy equation can be written as

$$
\begin{aligned}
\frac{\mathrm{d}}{\mathrm{d} t} E_{\text {kin }} & =\frac{1}{2} \frac{\mathrm{d}}{\mathrm{d} t} \int_{V} u_{\mathrm{p}}^{2} \mathrm{~d} V \\
& =-D_{\text {kin }}+M_{z}+M_{\varphi}+I_{\text {kin }}-G r I_{\mathrm{T}}-H a^{2} I_{\mathrm{L}}
\end{aligned}
$$

$D_{\text {kin }}$ represents the rate of viscous dissipation

$$
D_{\text {kin }}=\int_{V}\left(\nabla \times \boldsymbol{u}_{\mathrm{p}}\right)^{2} \mathrm{~d} V-2 \int_{S}\left(\frac{v_{\mathrm{p}}^{2}}{r}\right)_{r=1 / \Gamma} \mathrm{d} S,
$$

where $S$ is the free surface of the volume. $M_{z}$ and $M_{\varphi}$ denote the work done by thermocapillary forces acting on the cylindrical surface in the axial and azimuthal directions, respectively:

$$
\begin{gathered}
M_{z}=\int_{S}\left(w_{\mathrm{p}} \partial_{r} w_{\mathrm{p}}\right)_{r=1 / \Gamma} \mathrm{d} S, \\
M_{\varphi}=\int_{S} v_{\mathrm{p}}\left(\partial_{r} v_{\mathrm{p}}-\frac{v_{\mathrm{p}}}{r}\right)_{r=1 / \Gamma} \mathrm{d} S .
\end{gathered}
$$

$I_{\text {kin }}$ contains integrals resulting from interactions between basic state and disturbance flow:

$$
\begin{aligned}
I_{\text {kin }} & =I_{\text {kin }, 1}+I_{\text {kin }, 2}+I_{\text {kin }, 3}+I_{\text {kin }, 4}+I_{\text {kin }, 5} \\
& =-\int_{V}\left(v_{\mathrm{p}}^{2} \frac{u_{0}}{r}+u_{\mathrm{p}}^{2} \partial_{r} u_{0}+u_{\mathrm{p}} w_{\mathrm{p}} \partial_{z} u_{0}+w_{\mathrm{p}} u_{\mathrm{p}} \partial_{r} w_{0}+w_{\mathrm{p}}^{2} \partial_{z} w_{0}\right) \mathrm{d} V .
\end{aligned}
$$

$I_{\mathrm{T}}$ is the work done by buoyancy forces and reads

$$
I_{\mathrm{T}}=-\int_{V} \theta_{\mathrm{p}} w_{\mathrm{p}} \mathrm{d} V
$$

Finally, $I_{\mathrm{L}}$ denotes the work done by Lorentz forces. In order to elucidate the effect of the electrical potential $\phi$ we split $I_{\mathrm{L}}$ into two terms $I_{\mathrm{L}}=I_{\mathrm{L}, 1}+I_{\mathrm{L}, 2}$, where

$$
I_{\mathrm{L}, 1}=\int_{V}\left(u_{\mathrm{p}}^{2}+v_{\mathrm{p}}^{2}\right) \mathrm{d} V
$$




$$
I_{\mathrm{L}, 2}=\int_{V}\left(\frac{u_{\mathrm{p}}}{r} \partial_{\varphi}-v_{\mathrm{p}} \partial_{r}\right) \phi_{\mathrm{p}} \mathrm{d} V .
$$

If $\alpha>0$ the rate of change of energy is positive and the basic flow is unstable. Accordingly, positive/negative terms on the right-hand side of (42) have a net destabilizing/stabilizing effect. Similarly, a balance for the thermal energy transfer can be obtained by multiplying (35) by $\theta_{\mathrm{p}}$ followed by a volume integration. The thermal balance is the same as for the corresponding non-magnetic problem (Wanschura et al. 1995) and plays virtually no role for low Prandtl number fluids which are investigated in the present study. For a detailed description of the thermal energy equations and the numerical methods applied to compute the integrals we refer to Wanschura et al. (1995). Since the energy equation (42) must be exactly satisfied by any solution of the linear stability problem, the relative error in the kinetic energy balance is defined as the residual normalized by the largest absolute value of the integrals on the right-hand side of (42):

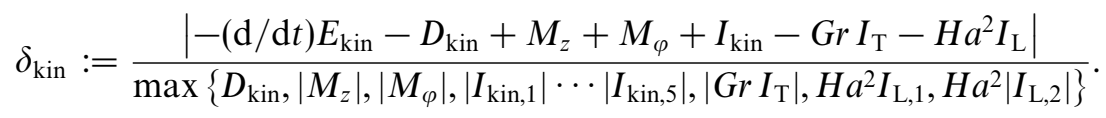

\section{Results}

\subsection{Influence of magnetic fields on the basic flow}

The axisymmetric thermocapillary basic flow in half-zones without magnetic fields is well-known from experimental (e.g. Preisser, Schwabe \& Scharmann 1983), numerical (e.g. Shen et al. 1990; Kuhlmann \& Rath 1993; Wanschura et al. 1995) and analytical (e.g. Kuhlmann 1989) studies. Near the free surface a steady toroidal vortex arises, the radial extent of which is approximately equal to the half-zone's height if the Reynolds number is large and $\Gamma \leqslant 1$. For small aspect ratios $\Gamma$ the flow pattern includes additional exponentially weak vortex rings inside the bulk. In the limit $R e \rightarrow 0, P r \rightarrow 0$ the streamlines are symmetric with respect to the midplane $z=0$. This symmetry is lost for increased Reynolds or Prandtl numbers due to nonlinear processes.

Figure 2 shows streamlines for $B i=G r=0, P r=0.02, \Gamma=1, \operatorname{Re}=2061$ and various Hartmann numbers $(\mathrm{Ha}=0,15,30,50)$ with a grid resolution of 30 collocation points in radial direction and 80 axial finite-difference steps. The state is neutrally stable for $H a=0$ as discussed in the next section. Owing to the damping effect of Lorentz forces axial magnetic fields reduce the volume transport of the thermocapillary vortex which is given by the maximum value $\Psi_{\max }$ of the Stokes stream function (figure 3). The symmetry with respect to the midplane is partially restored for increasing Hartmann numbers due to the diminution of convective (i.e. nonlinear) terms. Since Lorentz forces are purely radial (see §3.1) the flow tends to avoid radial momentum. As a result, the radial extent of the thermocapillary vortex decreases with increasing Hartmann number, leading to the formation of secondary vortices inside the bulk. These secondary vortices are, however, at least two orders of magnitude weaker than the primary vortex and can be neglected. Figure 4 reveals that the width of the thermocapillary vortex $\delta_{\|}$is approximately proportional to $\mathrm{Ha}^{-1 / 2}$ for sufficiently large Hartmann numbers $\left(\delta_{\|}\right.$is defined as the radial position where $\Psi=0$ on the midplane $z=0$ ). A $H^{-1 / 2}$-scaling is a typical feature for boundary layers which develop parallel to magnetic field lines (see e.g. Moreau 1990). We find that the surface velocity also scales with $\mathrm{Ha}^{-1 / 2}$ if the Hartmann number exceeds 150 (figure 4). 

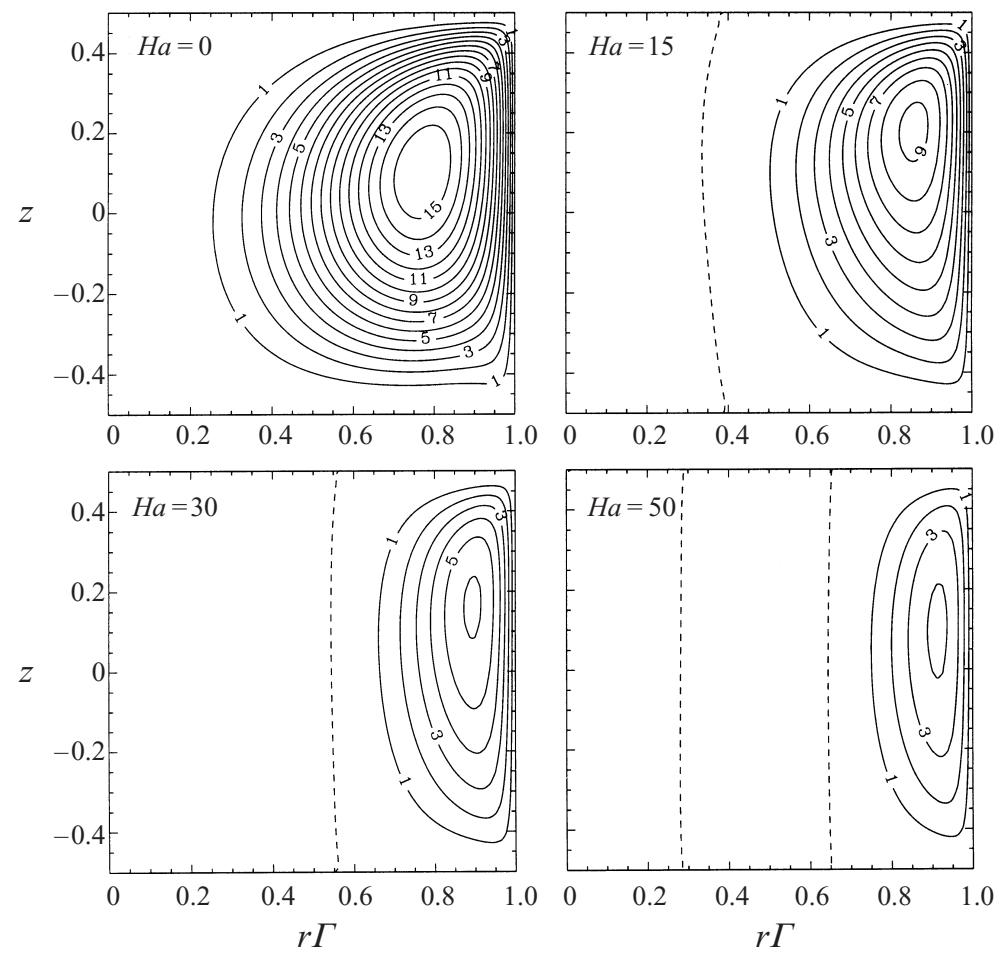

Figure 2. Streamlines (lines of constant $\Psi$ ) for the basic state $B i=G r=0, \operatorname{Pr}=0.02, \Gamma=1$, $R e=2061$ and various Hartmann numbers. Dashed lines represent $\Psi=0$.

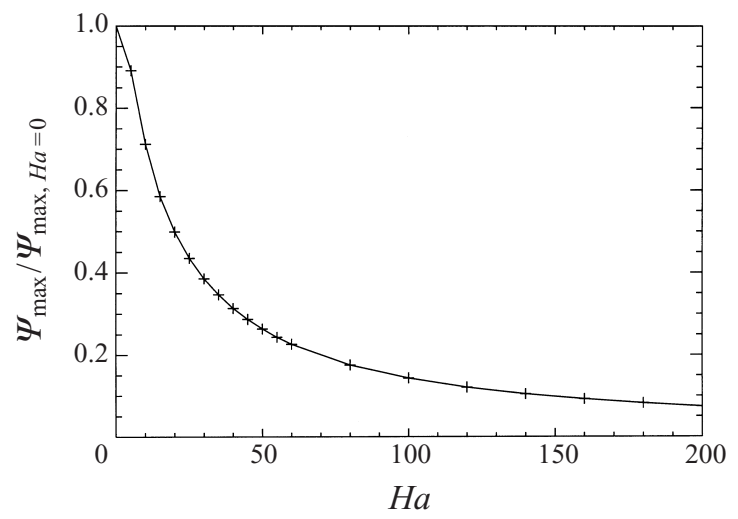

Figure 3. Dependence of the volume transport (maximum $\Psi$ ) on $\mathrm{Ha}$ for $\mathrm{Bi}=\mathrm{Gr}=0, \mathrm{Pr}=0.02$, $\Gamma=1, R e=2061$.

4.2. Flow stabilization by axial magnetic fields

The basic state for $B i=G r=0, P r=0.02, \Gamma=1$ at $R e=2061$ is neutrally stable with respect to the most dangerous mode $m=2$ if magnetic fields are absent $(\mathrm{Ha}=0)$, i.e. $R e_{\mathrm{c}}=R e_{\mathrm{c}}^{(m=2)}=2061$. The bifurcation is stationary, i.e. $\omega=0$, and the instability mechanism has been described in detail by Wanschura et al. (1995). By analysing the energy balance they showed that the integral $I_{\mathrm{kin}, 4}$ increases strongly with $R e$. $I_{\text {kin, } 4}$ measures the transfer of axial base-state momentum $w_{0}$ to the disturbance $w_{\mathrm{p}}$ 


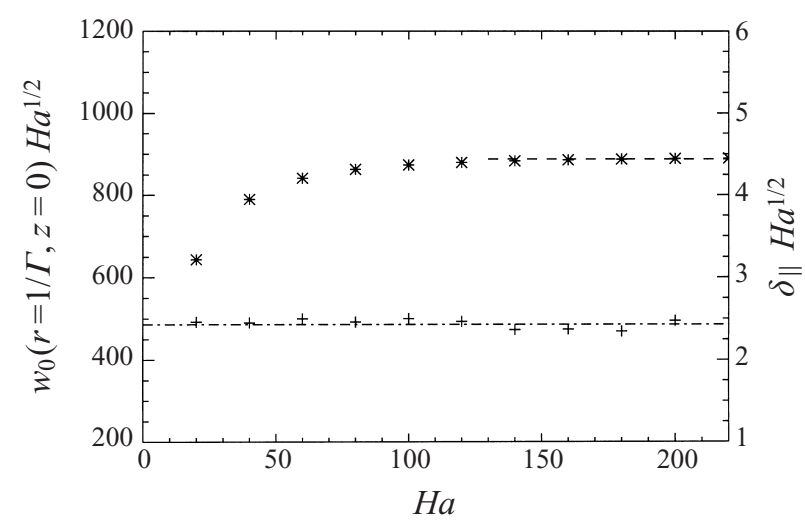

FIGURE 4. Dependence of $\delta_{\|} H a^{1 / 2}$ (crosses) and $w_{0}(r=1 / \Gamma, z=0) H a^{1 / 2}$ (asterisks) on $H a$ for $B i=G r=0, \operatorname{Pr}=0.02, \Gamma=1, \operatorname{Re}=2061$. The dashed-dotted line corresponds to a linear relation between the width of the thermocapillary vortex $\delta_{\|}$and $H \mathrm{a}^{-1 / 2}$, while the dashed line corresponds to a linear relation between the basic-state surface velocity and $H a^{-1 / 2}$.

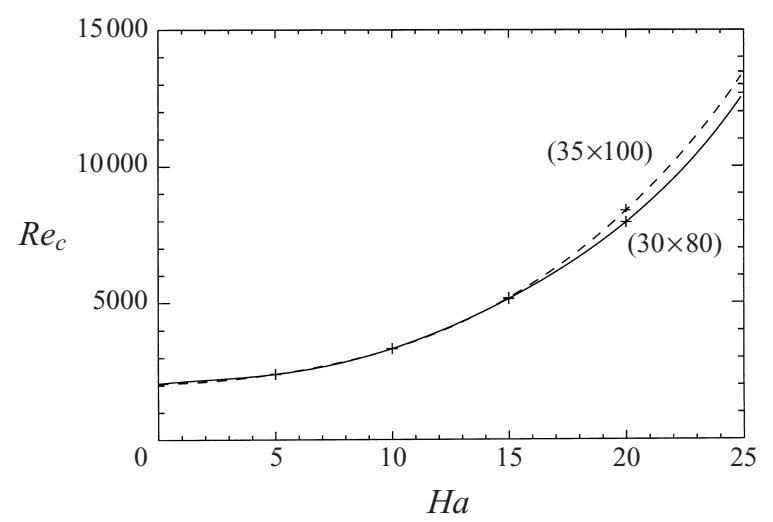

FIGURE 5. Dependence of the critical Reynolds number $\operatorname{Re}_{c}$ on $\mathrm{Ha}$ for $\mathrm{Bi}=\mathrm{Gr}=0, \mathrm{Pr}=0.02$, $\Gamma=1$. The azimuthal wavenumber of the critical mode is $m=2$. Solid line: $(30 \times 80)$-grid. Dashed line: $(35 \times 100)$-grid. The curves represent neutral stability of the basic state.

via the radial disturbance $u_{\mathrm{p}}$. This process intensifies with an increase of the radial gradient of base-state axial velocity. Hence, it follows that the low Prandtl number $(P r \ll 1)$ instability is due to the large strain rate present in the basic axial shear flow. Up to now, the stationary instability in small Prandtl number fluids was only observed in numerical studies (Rupp et al. 1989; Kuhlmann \& Rath 1993; Levenstam \& Amberg 1995; Wanschura et al. 1995). Experimental evidence is still missing since model experiments are usually carried out with large Prandtl number liquids (e.g. $\mathrm{NaNO}_{3}, \mathrm{KCl}$, silicon oils) where the first instability is oscillatory (e.g. Preisser et al. 1983; Velten, Schwabe \& Scharmann 1991) and due to hydrothermal waves as discussed by Wanschura et al. (1995).

To study the stabilizing effect of axial magnetic fields we fix the set of parameters ( $B i=G r=0, P r=0.02, \Gamma=1)$ for the moment and calculate the dependence of $R e_{c}$ on Ha. The results for two different grid resolutions are shown in figure 5 and in table 1 . The stationary $m=2$ mode remains critical over the range of $\mathrm{Ha}$ considered. The divergence of the two curves in figure 5 for $\mathrm{Ha}>15$ indicates that 


\begin{tabular}{rccc}
\hline$H a$ & $R e_{c}(35 \times 100)$ & $R e_{c}(30 \times 80)$ & $\delta_{\text {kin }}(30 \times 80)$ \\
0 & 2060 & 2061 & $0.9 \%$ \\
5 & 2400 & 2400 & $1.4 \%$ \\
10 & 3331 & 3331 & $3.3 \%$ \\
15 & 5167 & 5141 & $8.9 \%$ \\
20 & 8375 & 7947 & $20.8 \%$ \\
25 & 13458 & 12682 & $33.3 \%$
\end{tabular}

TABLE 1. Dependence of $\operatorname{Re}_{c}$ on $H a(B i=G r=0, \operatorname{Pr}=0.02, \Gamma=1, m=2)$ for two different grid resolutions. The relative error in the kinetic energy balance is given in the right-hand column for the $(30 \times 80)$-grid.

$\begin{array}{cccc}\text { Grid } & R e_{c}(\mathrm{Ha}=10) & R e_{c}(\mathrm{Ha}=15) & R e_{c}(\mathrm{Ha}=20) \\ 15 \times 40 & 5192 & 3116 & 4776 \\ 20 \times 60 & 3552 & 4371 & 6121 \\ 25 \times 80 & 3357 & 5045 & 7123 \\ 30 \times 80 & 3331 & 5141 & 7947 \\ 35 \times 100 & 3331 & 5167 & 8375 \\ 40 \times 120 & 3331 & 5168 & 8432\end{array}$

TABLE 2. Dependence of $R e_{c}$ on the grid resolution for $H a=10, H a=15$ and $H a=20$

$(B i=G r=0, P r=0.02, \Gamma=1, m=2)$.

the grid resolution must be enhanced with increasing Hartmann number due to the development of the velocity boundary layer near the free surface. Numerous grid resolution tests were carried out. Table 2 shows some of the results and gives a feeling for the effect of grid size on the critical Reynolds number. For $H a=10$ a grid with 30 radial and 80 axial points is sufficient. In this case the relative error in the kinetic energy balance $\delta_{\text {kin }}$ is about 3\% (table 1). The critical Reynolds number for $\mathrm{Ha}=15$ calculated using the $(30 \times 80)$-grid differs by only $0.5 \%$ compared to the $(40 \times 120)$-grid computation. We can deduce, therefore, that the $(30 \times 80)$-grid is also appropriate for stability analyses with $H a=15$. Additionally, a very high resolution calculation with $50 \times 130$ points was performed which confirms the critical Reynolds number obtained by the $(40 \times 120)$-grid. For $H a>15$ higher grid resolutions are necessary to achieve grid convergence. With our present computational resources such calculations are not feasible. For $H a=20$ the values in table 2 suggest that insufficient grid resolutions result in critical Reynolds numbers which are too low. For the following analyses, we restrict the calculations to $H a \leqslant 15$ using a grid of $30 \times 80$ points. This ensures reliable results. Relative errors in the thermal energy balance are always below $0.2 \%$.

In order to understand the stabilizing mechanism of the magnetic field we consider the kinetic energy balance. Figure 6 shows the relevant integrals from (42) for varying Hartmann number and fixed parameters $B i=G r=0, P r=0.02, \Gamma=1, R e=3331$, $m=2$. For $H a=10$ the basic state is neutrally stable. As $I_{\text {kin,4 }}$ remains the dominant destabilizing term even for higher Hartmann numbers, we conclude that axial magnetic fields do not change the mechanism of instability, namely the instability of the axial shear flow. Also, it is obvious that the modification of the basic flow described above is only of minor importance for the magnetic stabilizing effect since $I_{\text {kin }}$ (normalized by $D_{\text {kin }}$ ) is almost invariant. Note that $I_{\text {kin }}$ is the only integral in (42) which includes basic-state quantities. The rise in $H a^{2} I_{\mathrm{L}}$ with increasing Hartmann number shows that the stabilization is primarily due to the direct action of the magnetic 


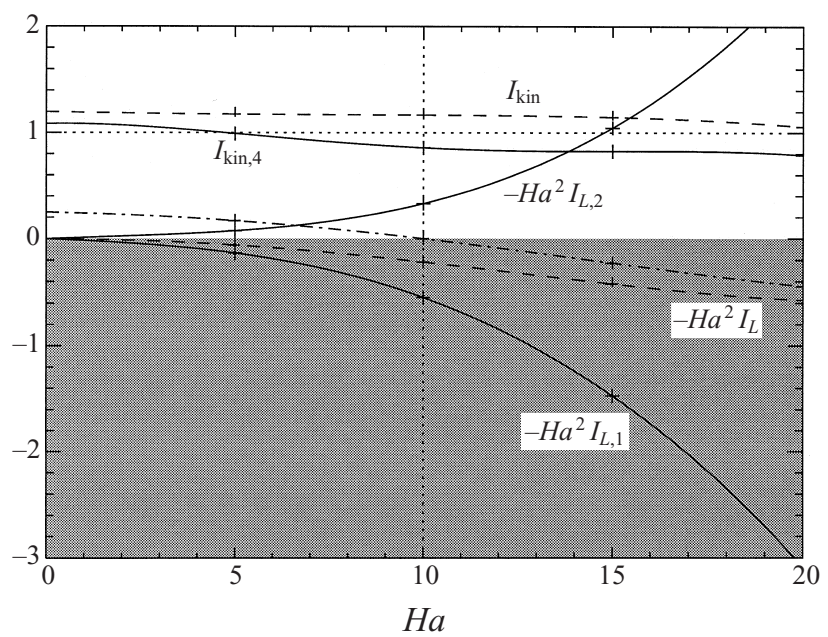

Figure 6. The rate of change of kinetic energy as a function of $\mathrm{Ha}$ for $\mathrm{Bi}=\mathrm{Gr}=0, \mathrm{Pr}=0.02$, $\Gamma=1, R e=3331$ and $m=2$. All terms are normalized with respect to $D_{\text {kin }}$. Dashed-dotted: $(\mathrm{d} / \mathrm{d} t) E_{\text {kin }}$. Dotted: $D_{\text {kin }}$. The vertical dotted line marks the stability boundary.

(a)

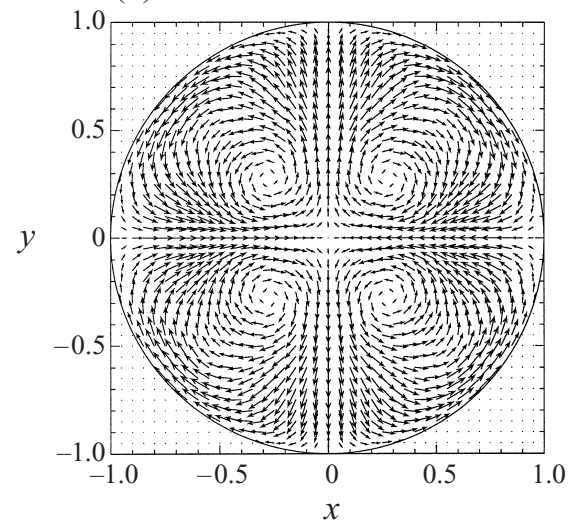

(b)

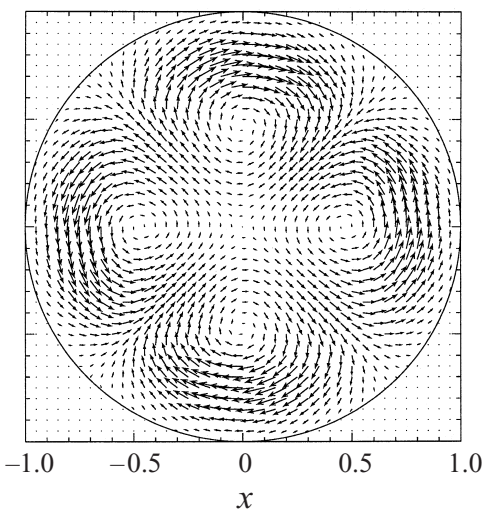

FIgURE 7. Horizontal cut at $z=0$ through the neutral mode $B i=G r=0, \operatorname{Pr}=0.02, \Gamma=1$, $R e=R e_{c}=3331, H a=10, m=2$ : Flow field $(a)$ and electrical currents $(b)$.

field on the disturbance flow. Induced electric currents instantly give rise to damping Lorentz forces. $H a^{2} I_{\mathrm{L}}$ consists of the positive (and thus always stabilizing) $I_{\mathrm{L}, 1}$ and the negative $I_{\mathrm{L}, 2}$. The latter includes the effects of induced electric fields and strongly reduces the stabilizing action of electromagnetic forces. Figure 7 shows a horizontal cut through the neutral fields of velocity and electric current density. The induced electric field allows electric currents which are not perpendicular to the perturbation flow. Accordingly, the damping effect of Lorentz forces is reduced. The induction of electric fields is necessary to ensure the conservation of electric charge. Therefore, neglecting electric fields in three-dimensional fluid flow as in Baumgartl et al. (1990) is not justified and results in an overestimation of magnetic stabilization.

\subsection{Variation of the aspect ratio}

Both experimental (Preisser et al. 1983) and numerical studies (Wanschura et al. 1995 ) pointed out that the critical wavenumber $m$ is roughly proportional to the 


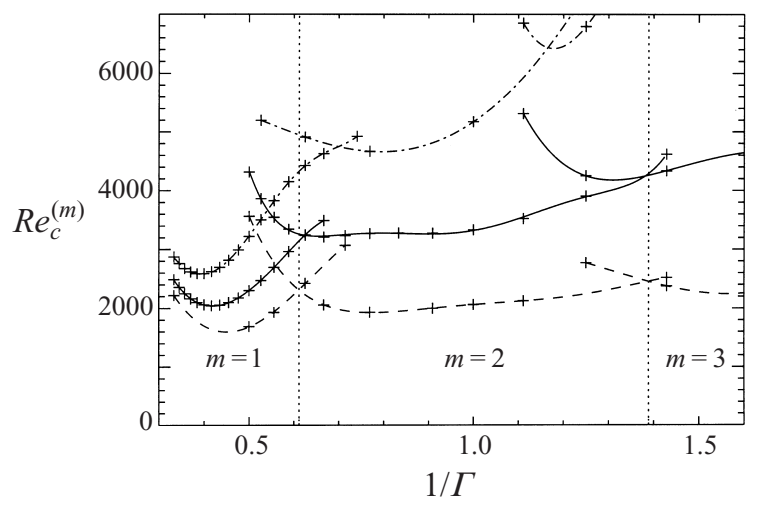

FiguRE 8. Dependence of critical Reynolds numbers and wavenumbers on $1 / \Gamma$ for $B i=G r=0$, $P r=0.02$. Dashed lines: $H a=0$. Solid lines: $H a=10$. Dashed-dotted lines: $H a=15$. The vertical dotted lines mark the changes of the critical mode for $H a=0$.

(a)

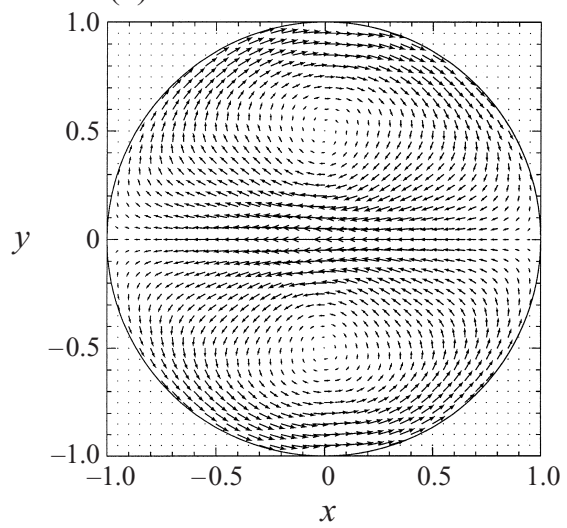

(b)

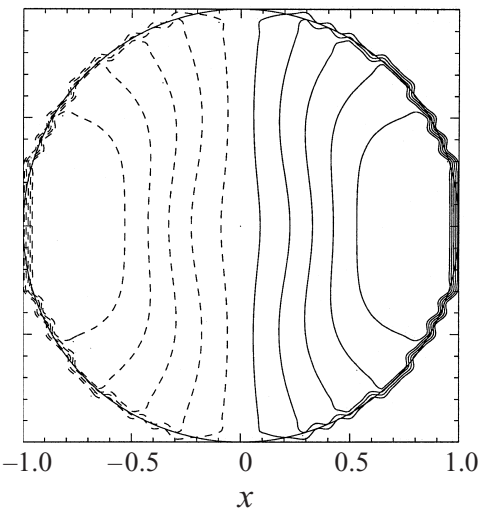

FiguRE 9. Disturbance flow $(a)$ and temperature field $(b)$ of the critical mode in a horizontal cross-section at $z=0$ for $B i=G r=0, P r=0.02,1 / \Gamma=0.5, H a=15, R e=R e_{c}=3221$ and $m=1$. Solid contour lines indicate positive values (warm), dashed contours indicate negative values (cold). (Note that the waviness of the isolines close to the free surface is an artefact of the visualization software.)

inverse aspect ratio $1 / \Gamma$ of the half-zone since the azimuthal wavelength scales with the characteristic length $d$. As described in $\S 4.1$ axial magnetic fields introduce a new, smaller length scale by reducing the radial extent of the thermocapillary flow. Thus, we would expect a tendency toward larger critical wavenumbers with increasing Hartmann number. Figure 8 shows the dependence of critical Reynolds numbers and wavenumbers on $1 / \Gamma$ for $B i=G r=0, P r=0.02$ and various Hartmann numbers. The stabilizing influence of Lorentz forces strengthens with increasing $1 / \Gamma$. Concerning the azimuthal symmetry of the critical modes, magnetic fields actually favour modes with larger wavenumbers if $1 / \Gamma>1$ as can be seen by the shift of the intersection of curves for different wavenumbers. However, for $1 / \Gamma<1$ we observe an opposite effect, i.e. axial magnetic fields favour $m=1$ above $m=2$. This can be attributed to a stabilizing effect of azimuthal thermocapillary forces. 


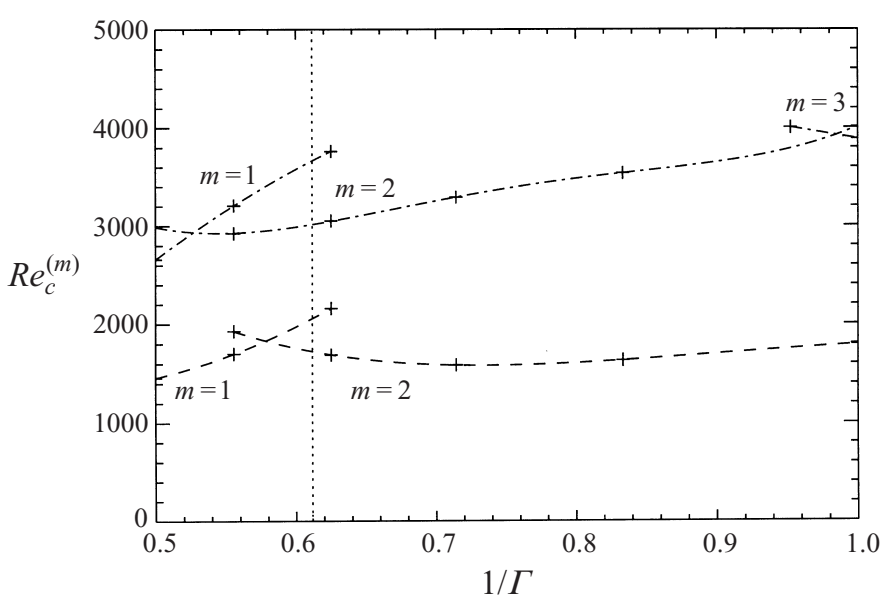

FIGURE 10. Dependence of critical Reynolds numbers and wavenumbers on $1 / \Gamma$ for $B i=G r=0$, $\operatorname{Pr}=10^{-5}$. Dashed lines: $H a=0$. Dashed-dotted lines: $H a=15$. The vertical dotted line marks the change of the critical mode for $H a=0$ and $P r=0.02$ at $1 / \Gamma=0.61$.

To illustrate this effect figure 9 shows horizontal cross-sections of the neutral fields of velocity and temperature for $B i=G r=0, P r=0.02,1 / \Gamma=0.5, H a=15$, $R e=R e_{c}=3221, m=1$. The temperature distribution arises mainly due to axial convection of the conductive temperature profile induced by the disturbance flow (see Wanschura et al. 1995). The azimuthal temperature gradients at the free surface cause thermocapillary forces which counteract the surface flow, thus damping the perturbation. Although this effect occurs for any mode of disturbance, its efficiency depends on the wavenumber. Short-wave modes are damped more effectively than modes with larger wavelengths since thermocapillary forces are proportional to azimuthal surface temperature gradients which scale with $m$ according to (37). Also, the strength of thermocapillary forces increases with Re. Due to magnetic stabilization the critical Reynolds numbers increase such that thermocapillary stabilization intensifies. This in turn causes a further increase of $R e_{c}$. Therefore, azimuthal thermocapillary forces amplify magnetic stabilization and favour small wavenumbers.

For $\mathrm{Pr} \rightarrow 0$ azimuthal thermocapillary forces vanish, since heat diffusion prevents the occurrence of temperature perturbations. Consequently, we observe a general tendency toward larger critical wavenumbers with increasing Ha (figure 10). The effect of $\mathrm{Pr}$ is studied in detail in the following.

\subsection{Variation of the Prandtl number}

Prandtl numbers of semiconductor melts are of the same order of magnitude as those of liquid metals (e.g. $\mathrm{Si}: P r=0.02$; Ga: $P r=0.02$; GaAs: $P r=0.07$ ). Figure 11 shows the dependence of critical Reynolds numbers and wavenumbers on $\operatorname{Pr}$ for $B i=G r=0, \Gamma=1$ and various Hartmann numbers. Our value of $R e_{c}=1899$ for $\operatorname{Pr}=0.01$ without magnetic field is in excellent agreement with the result of Levenstam \& Amberg (1995) who found $R e_{c}=1960$.

In the limit $P r \rightarrow 0$ the critical wavenumber increases with the Hartmann number as described in the previous section. With growing Prandtl number convective heat transport increases, resulting in intensified azimuthal temperature gradients leading to 


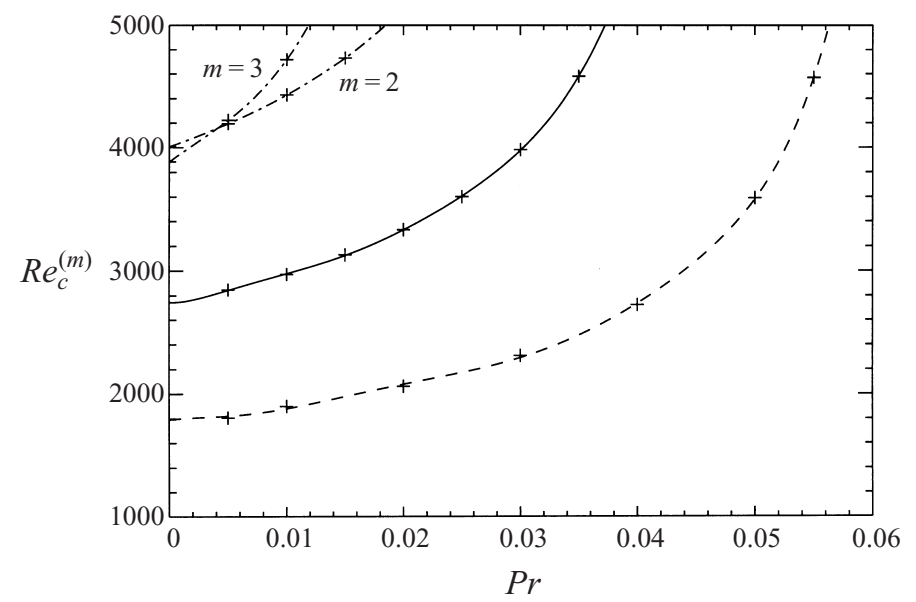

FIGURE 11. Dependence of critical Reynolds numbers on $\operatorname{Pr}$ for $B i=G r=0, \Gamma=1$ and various $H a$. Dashed line: $R e_{c}^{(m=2)}=R e_{c}$ for $H a=0$. Solid line: $R e_{c}^{(m=2)}=R e_{c}$ for $H a=10$. Dashed-dotted lines: $\operatorname{Re}_{c}^{(m=3)}$ and $R e_{c}^{(m=2)}$ for $H a=15$.

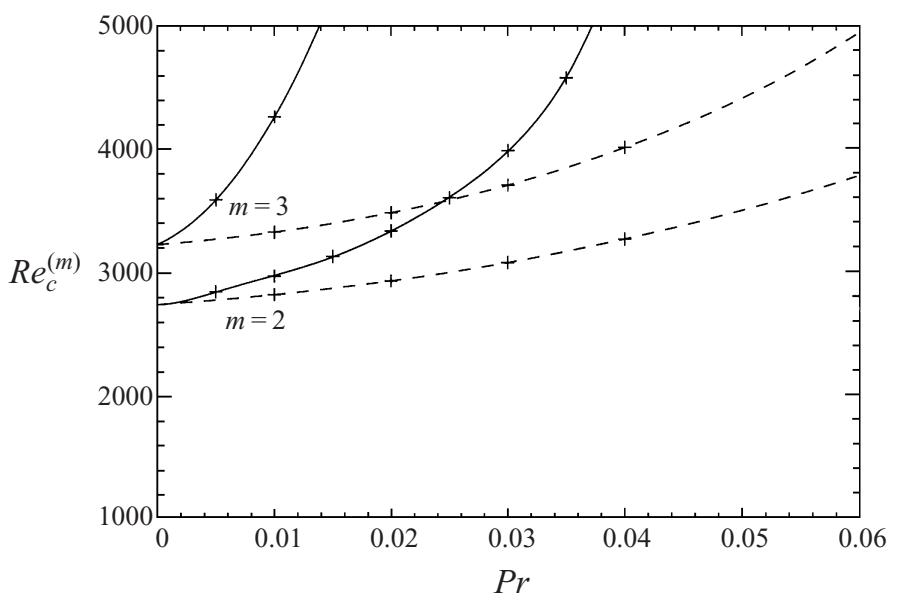

FIGURE 12. Dependence of $\operatorname{Re}_{c}^{(m)}$ on $P r$ for $B i=G r=0, \Gamma=1, H a=10$ and $m=2$, 3. Solid lines: with conventional disturbance thermocapillary boundary conditions (A 8) and (A 9). Dashed lines: without azimuthal thermocapillary forces, i.e. $R e=0$ in (A9).

thermocapillary forces which counteract the disturbance surface flow. As a result, the flow is stabilized and the critical Reynolds number increases accordingly. Since the damping effect of azimuthal thermocapillary forces on the disturbance flow increases with $\mathrm{m}$, the most dangerous mode for $\mathrm{Ha}=15$ changes as $\mathrm{Pr}$ increases. Figure 12 illustrates this effect for $B i=G r=0, \Gamma=1, H a=10$. The dashed lines represent the dependence of critical Reynolds numbers on $\mathrm{Pr}$ when azimuthal thermocapillary forces are neglected by artificially setting $R e=0$ in the azimuthal thermocapillary boundary condition (A 9). In this case there is only a small stabilization with increasing Prandtl number. The solid lines take account of azimuthal thermocapillary forces and we observe a considerable stabilization whose effectiveness increases with $m$. Figure 12 clearly shows that the main reason for the critical Reynolds number dependence on $\mathrm{Pr}$ is the stabilizing effect of azimuthal thermocapillary forces rather than a modified basic flow as suggested by Wanschura et al. (1995). 


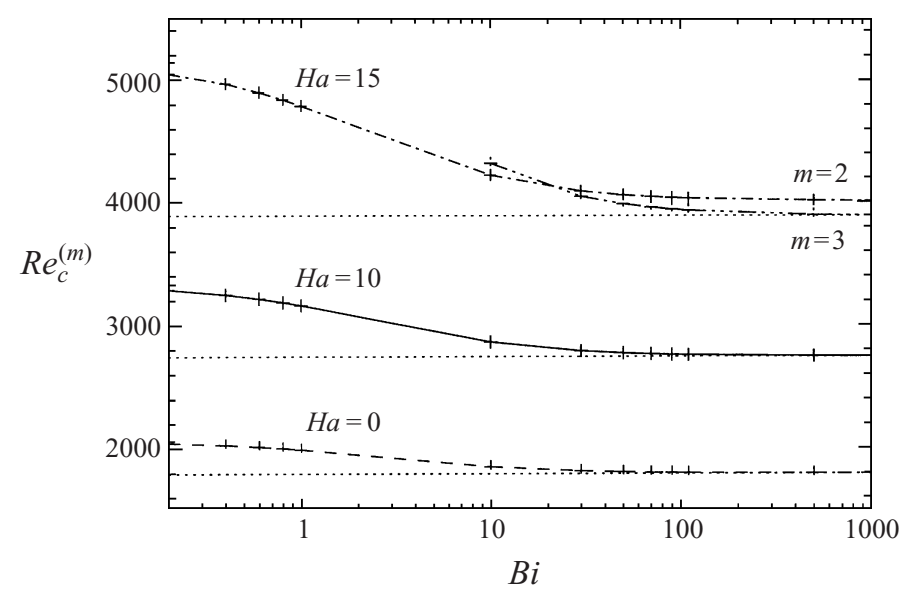

Figure 13. Dependence of $\operatorname{Re}_{c}^{(m)}$ on $B i$ for $G r=0, \operatorname{Pr}=0.02, \Gamma=1$ and various $H a$. Dashed line: $R e_{c}^{(m=2)}=R e_{c}$ for $H a=0$. Solid line: $R e_{c}^{(m=2)}=R e_{c}$ for $H a=10$. Dashed-dotted lines: $R e_{c}^{(m=2)}$ and $R e_{c}^{(m=3)}$ for $H a=15$. The dotted lines mark the critical Reynolds numbers $\operatorname{Re}_{c}$ for $B i=G r=0$, $\Gamma=1$ and $\mathrm{Pr} \rightarrow 0$ for the corresponding Hartmann numbers $H a=0,10,15$.

We emphasize that the increase of $R e_{c}$ shown in figure 11 is limited. For sufficiently large Prandtl numbers $(\mathrm{Pr}>0.5)$ the basic flow becomes linearly unstable to a pair of hydrothermal waves propagating nearly azimuthally which result from a synchronous coupling of conductive and convective heat transport (Kuhlmann \& Rath 1993; Wanschura et al. 1995). For semiconductor melts $(P r \ll 1)$ the hydrothermal wave instability mechanism plays no role.

\subsection{Variation of the Biot number}

The Biot number determines the heat transfer rate from the free surface to the surroundings according to (13). Figure 13 presents critical Reynolds numbers as a function of $B i$ for $G r=0, P r=0.02, \Gamma=1$ and various Hartmann numbers. In the limit $B i \rightarrow \infty$ no surface temperature perturbations can arise and the stability boundaries coincide with the case $\mathrm{Pr} \rightarrow 0$. With decreasing $B i$ azimuthal thermocapillary forces stabilize the basic flow and cause a change of the most dangerous mode for $H a=15$.

\subsection{The effect of buoyancy}

Within the Boussinesq approximation the ratio $\mathrm{Gr} / \mathrm{Re}$ is a constant with regard to a given geometry:

$$
\frac{G r}{\operatorname{Re}}=\frac{\beta g \rho_{0} d^{2}}{\gamma} .
$$

As an example, we consider a $S i$ liquid bridge with $d=10^{-2} \mathrm{~m}$. Under terrestrial gravity conditions the corresponding ratio $\mathrm{Gr} / \mathrm{Re}$ is approximately 1.25 . Figure 14 shows the dependence of $R e_{c}$ on $\mathrm{Ha}$ considering the influence of buoyancy forces. If the liquid bridge is heated from below $(G r=1.25 R e)$ the effect of buoyancy is destabilizing. Heating from above $(G r=-1.25 R e)$ stabilizes the basic state. Since $G r \sim R e$ the effect of buoyancy on stability increases with the critical Reynolds number and thus with $\mathrm{Ha}$.

The destabilizing action of buoyancy when heating the liquid bridge from below is due to radial temperature gradients in both the basic state and the disturbance. The basic flow vortex (figure 2) transports warm liquid from the bottom to the free surface 


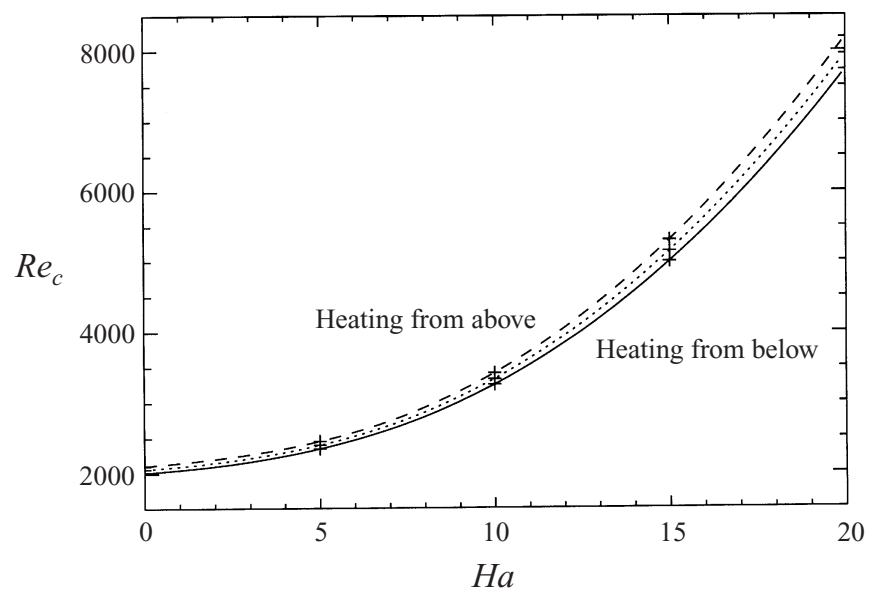

Figure 14. Dependence of $\operatorname{Re}_{c}$ on $H a$ for $B i=0, \operatorname{Pr}=0.02, \Gamma=1$ in the presence of buoyancy forces. Dashed line: $G r=-1.25 R e$ (heating from above). Solid line: $G r=1.25 R e$ (heating from below). Dotted line: $G r=0$. The critical wavenumber is $m=2$ in all cases.

and cold liquid into the interior. The resulting radial temperature gradients cause buoyancy forces which strengthen the basic vortex and thus favour instability. When heating from above the presence of radial temperature gradients has a stabilizing effect. The influence of buoyancy on stability increases with $\mathrm{Pr}$ owing to increased temperature gradients caused by intensified convective heat transport.

In this study buoyancy is considered only as a disturbing effect for the thermocapillary flow. We investigated the instability of a unique basic state which is dominated by thermocapillary forces. However, if $\mathrm{Gr}$ exceeded a critical value, the onset of RayleighBénard convection would give rise to multiple basic states where the various flow patterns were characterized by the mutual orientation of buoyancy- and thermocapillaryinduced vortices. For the stability of combined buoyant-thermocapillary flow in liquid bridges the reader is refered to Wanschura, Kuhlmann \& Rath (1997).

\section{Discussion}

The stabilizing effect of steady axial magnetic fields on the two-dimensional thermocapillary flow in half-zones has been investigated for a wide range of parameters. The half-zone model represents a simplification of the floating-zone configuration used for crystal growth.

Axial magnetic fields cause a concentration of the thermocapillary convection near the free surface. This was also observed in the floating-zone simulation of Morthland \& Walker (1996) and confirmed experimentally by Cröll et al. (1994). The latter authors examined doped $\mathrm{Si}$ crystals grown with the floating-zone technique $\left(R e \approx 2 \times 10^{5}\right)$ under the influence of a steady axial magnetic field $(H a \approx 185)$ and found a strong radial variation of the dopant concentration. They attributed the radial macro-segregation to a flow pattern which is characterized by intense convection near the free surface and fluid nearly at rest in the interior of the melt zone.

If the thermocapillary Reynolds number exceeds a critical value, the twodimensional basic state becomes unstable and a transition to three-dimensional flow takes place. For the low Prandtl number fluids considered in this study (liquid metals and semiconductor melts) this bifurcation is stationary and due to a shear flow 
instability. As discussed by Wanschura et al. (1995) the confinement of the flow by boundaries is essential for the instability to occur. Thus, model studies with infinite geometries, like e.g. Priede \& Gerbeth (1995) and Priede et al. (1994), are not applicable to the float-zone geometry. Experimental evidence for the stationary instability in low Prandtl number liquid bridges is still missing. Results of model experiments carried out with large Prandtl number fluids are not applicable to low Prandtl number fluids due to completely different instability mechanisms.

By an energy analysis we have shown that the stabilizing effect of magnetic fields on thermocapillary flows in liquid bridges is mainly due to the direct action of Lorentz forces on the disturbance flow. The modification of the basic flow by magnetic fields is of minor importance. Induced electric fields, however, reduce the magnetic stabilizing effect considerably.

Azimuthal thermocapillary forces have an additional stabilizing influence by damping the disturbance flow. This effect increases with $R e$ and thus with $H a$ since magnetic fields enhance the critical Reynolds number. The stabilizing effects of azimuthal thermocapillary forces and magnetic fields amplify each other. Moreover, the azimuthal thermocapillary stabilization depends on the wavenumber $m$ such that short-wave modes are damped more strongly than those with larger wavelengths.

The results clearly show that the degree of magnetic stabilization depends on $\Gamma$, $P r, B i$ and $G r$. The stabilization intensifies with decreasing aspect ratio, increasing Prandtl number, decreasing Biot number and/or decreasing Grashof number.

As in previous studies without magnetic field (Rupp et al. 1989; Kuhlmann \& Rath 1993; Levenstam \& Amberg 1995) no critical axisymmetric (i.e. $m=0$ ) mode was found despite extensive parameter variations. The application of an axial magnetic field does not alter the fact that the first instability of thermocapillary flow in halfzones is axisymmetry-breaking. For instance, with the parameters $\mathrm{Bi}=\mathrm{Gr}=0, \mathrm{Pr}=$ $0.02, \Gamma=1$ and $H a=20$ we carried out stability calculations for the axisymmetric mode and found no positive growth rate for Reynolds numbers up to 40000 where we finally terminated the computations. Thus, stability analyses of thermocapillary convection in liquid bridges which consider axisymmetric perturbations solely, with or without magnetic fields (e.g. Shen et al. 1990; Chen \& Chin 1995), must be interpreted with the utmost caution.

The onset of time-dependent flow in floating zones is of particular practical interest since unsteady convection can cause undesirable spatial fluctuations of the dopant concentration (dopant striations) in growing crystals (Eyer \& Leiste 1985; Cröll et al. 1989, 1991). Time-dependent flow, however, occurs only after a second bifurcation (i.e. the instability of the non-axisymmetric steady state) and was not considered in the present study. Levenstam \& Amberg (1995) found the transition to the unsteady state to take place at $R e=6250$ for $P r=0.01$ and unit aspect ratio. Values given by Rupp et al. (1989) for aspect ratio $\Gamma=1.2$ are higher. They range from 14286 for $P r=0.007$ to 26471 for $P r=0.068$. The order of magnitude was confirmed by experiments of Cröll et al. (1989). It is interesting to note that not only is the stationary first instability suppressed with increasing Prandtl number, but also the onset of time-dependent flow. In the full-zone simulation of Baumgartl et al. (1990) the transition to unsteady thermocapillary convection occurs at much lower values, namely $R e=5750$ for $\operatorname{Pr}=0.02$ and $R e=8824$ for $P r=0.068$. These results indicate that the effect of $P r$ on the flow stability in full-zones is not as strong as in half-zones owing to different thermal boundary conditions. Unfortunately, Baumgartl et al. (1990) investigated the effect of static magnetic fields only for a relatively large Prandtl number of $\mathrm{Pr}=0.068$. Therefore, a quantitative comparison of their results with our half-zone study is not possible. 


\section{Outlook}

Numerical investigations using relatively simple models like the half-zone can be used to get insight into the basic physical mechanisms which arise in floating zones. A thorough understanding of these phenomena is required in order to develop efficient strategies to optimize the melt convection in real crystal growth configurations. Magnetic fields play a pivotal role in semiconductor crystal growth since they allow a contactless control of the melt flow due to the high electrical conductivity of the molten material. Improved dopant and defect distributions can thus be achieved.

Numerous applications of magnetic fields in floating-zone crystal growth are conceivable and must be examined in future investigations. The effects of cusped (see e.g. Baumgartl et al. 1990) and transverse (Kimura et al. 1983) magnetic fields are still rather unexplored. Also, the use of rotating magnetic fields appears to be a very promising method to suppress convective instabilities in semiconductor melt flow (e.g. Gelfgat 1995). The simultaneous application of static and rotating fields allows the benefits of both kinds of magnetic fields and will probably be the subject of many future experimental and numerical studies.

We are indebted to V. M. Shevtsova for providing the code for the energy balance computations.

\section{Appendix. Linear stability equations}

The linear system of partial differential equations for the three-dimensional stability problem reads:

$$
\begin{gathered}
-\left(u_{0} \partial_{r}+w_{0} \partial_{z}\right) u^{\prime}-\left(u^{\prime} \partial_{r}+w^{\prime} \partial_{z}\right) u_{0}-\partial_{r} p^{\prime}+\left(\mathrm{D} \partial_{r}-\frac{m^{2}}{r^{2}}+\partial_{z}^{2}\right) u^{\prime} \\
-\frac{u^{\prime}}{r^{2}}+\frac{2}{r}\left(\mathrm{D} u^{\prime}+\partial_{z} w^{\prime}\right)+H a^{2}\left(-u^{\prime}-\frac{m}{r} \mathrm{i} \phi^{\prime}\right)=\tilde{\alpha} u^{\prime}, \\
-\left(u_{0} \partial_{r}+w_{0} \partial_{z}\right) w^{\prime}-\left(u^{\prime} \partial_{r}+w^{\prime} \partial_{z}\right) w_{0}-\partial_{z} p^{\prime}+\left(\mathrm{D} \partial_{r}-\frac{m^{2}}{r^{2}}+\partial_{z}^{2}\right) w^{\prime}+G r \theta^{\prime}=\tilde{\alpha} w^{\prime}, \\
2\left\{\left[\left(\partial_{r}-\frac{1}{r}\right) u_{0} \partial_{r}+\partial_{r} w_{0} \partial_{z}\right] u^{\prime}+\left[\partial_{z} u_{0} \partial_{r}+\left(\partial_{z} w_{0}-\frac{1}{r} u_{0}\right) \partial_{z}\right] w^{\prime}\right\} \\
+\left(\mathrm{D} \partial_{r}-\frac{m^{2}}{r^{2}}+\partial_{z}^{2}\right) p^{\prime}-G r \partial_{z} \theta^{\prime}-H a^{2} \partial_{z} w^{\prime}=0, \\
-\left(u_{0} \partial_{r}+w_{0} \partial_{z}\right) \theta^{\prime}-\left(u^{\prime} \partial_{r}+w^{\prime} \partial_{z}\right) \theta_{0}+w^{\prime}+\frac{1}{P r}\left(\mathrm{D} \partial_{r}-\frac{m^{2}}{r^{2}}+\partial_{z}^{2}\right) \theta^{\prime}=\tilde{\alpha} \theta^{\prime}, \\
\left(\mathrm{D} \partial_{r}-\frac{m^{2}}{r^{2}}+\partial_{z}^{2}\right) \mathrm{i} \phi^{\prime}+\frac{1}{m}\left(r \partial_{r}+2\right)\left(\mathrm{D} u^{\prime}+\partial_{z} w^{\prime}\right)-\frac{m}{r} u^{\prime}=0 .
\end{gathered}
$$

The boundary conditions are

$$
u^{\prime}=w^{\prime}=\partial_{z} w^{\prime}=\theta^{\prime}=\partial_{z} \phi^{\prime}=0
$$

at $z=-\frac{1}{2}, \frac{1}{2}$, and

$$
\begin{gathered}
u^{\prime}=0, \\
\partial_{r} w^{\prime}+\operatorname{Re} \partial_{z} \theta^{\prime}=0,
\end{gathered}
$$




$$
\begin{gathered}
\frac{1}{\Gamma^{2}}\left(\partial_{r} \mathrm{D} u^{\prime}+\partial_{r} \partial_{z} w^{\prime}\right)+\operatorname{Re}^{2} \theta^{\prime}=0, \\
\left(\partial_{r}+B i\right) \theta^{\prime}=0, \\
\mathrm{i} \partial_{r} \phi^{\prime}+\frac{1}{m \Gamma}\left(\mathrm{D} u^{\prime}+\partial_{z} w^{\prime}\right)=0
\end{gathered}
$$

at $r=1 / \Gamma$. In addition, the solution must satisfy conditions of symmetry and regularity on the axis at $r=0$ (see e.g. Batchelor \& Gill 1962; Xu \& Davis 1984; Kuhlmann \& Rath 1993):

$$
\begin{gathered}
\partial_{r} u^{\prime}=w^{\prime}=p^{\prime}=\theta^{\prime}=\phi^{\prime}=0 \text { for } m=1, \\
u^{\prime}=w^{\prime}=p^{\prime}=\theta^{\prime}=\phi^{\prime}=0 \quad \text { for } m>1 .
\end{gathered}
$$

\section{REFERENCES}

Batchelor, G. K. \& Gill, A. E. 1962 Analysis of the stability of axisymmetric jets. J. Fluid Mech. 14, 529.

Baumgartl, J., Gewald, M., Rupp, R., Stierlen, J. \& Müller, G. 1990 The use of magnetic fields and microgravity in melt growth of semiconductors - a comparative study. Proc. 7th Eur. Symp. on Materials and Fluid Science in Microgravity, Oxford, ESA SP-295, p. 47.

Canuto, C., Hussaini, M. Y., Quarteroni, A. \& Zang, T. A. 1988 Spectral Methods in Fluid Dynamics. Springer.

Chandrasekhar, S. 1961 Hydrodynamic and Hydromagnetic Stability. Clarendon.

CHEN, J.-C. \& Chin, G.-H. 1995 Linear stability analysis of thermocapillary convection in the floating zone. J. Cryst. Growth 154, 98.

Cröll, A., Dold, P. \& Benz, K. W. 1994 Segregation in Si floating-zone crystals grown under microgravity and in a magnetic field. J. Cryst. Growth 137, 95.

Cröll, A., Müller-Sebert, W., Benz, K. W. \& Nitsche, R. 1991 Natural and thermocapillary convection in partially confined silicon melt zones. Micrograv. Sci. Technol. 3, 204.

Cröll, A., Müller-Sebert, W. \& Nitsche, R. 1989 The critical Marangoni number for the onset of time-dependent convection in silicon. Materials Res. Bull. 24, 995.

EyER, A. \& LeISTE, H. 1985 Striation-free silicon crystals by float-zoning with surface-coated melt. J. Cryst. Growth 71, 249.

GelfGAT, Y. M. 1995 Electromagnetic field application in the processes of single crystal growth under microgravity. Acta Astronaut. 37, 333.

Herrmann, F. M., Baumgartl, J., Feulner, T. \& Müller, G. 1992 The use of magnetic fields for damping unsteady Marangoni convection in GaAs floating zones under microgravity. Proc. 8th Eur. Symp. on Materials and Fluid Science in Microgravity, Brussels, ESA SP-333, p. 57.

Khine, Y. Y. \& WaLKer, J. S. 1994 Thermocapillary convection in a cylinder with a strong non-uniform axisymmetric magnetic field. J. Fluid Mech. 276, 369.

Kimura, H., Harvey, M. F., O’Connor, D. J., Robertson, G. D. \& Valley, G. C. 1983 Magnetic field effects on float-zone $\mathrm{Si}$ cryst. growth. J. Cryst. Growth 62, 523.

Kuhlmann, H. 1989 Small amplitude thermocapillary flow and surface deformations in a liquid bridge. Phys. Fluids A 1, 672

Kuhlmann, H. C. 1995 Some scaling aspects of thermocapillary flows. Microgravity Q. 1, 29.

Kuhlmann, H. C. \& Rath, H. J. 1993 Hydrodynamic instabilities in cylindrical thermocapillary liquid bridges. J. Fluid Mech. 247, 247.

Levenstam, M. \& AmberG, G. 1995 Hydrodynamical instabilities of thermocapillary flow in a half-zone. J. Fluid Mech. 297, 357.

Moreau, R. 1990 Magnetohydrodynamics. Kluwer.

Morthland, T. E. \& Walker, J. S. 1996 Thermocapillary convection during floating-zone silicon growth with a uniform or non-uniform magnetic field. J. Cryst. Growth 158, 471.

Preisser, F., Schwabe, D. \& Scharmann, A. 1983 Steady and oscillatory thermocapillary convection in liquid columns with free cylindrical surface. J. Fluid Mech. 126, 545. 
Press, W. H., Flannery, B. P., Teukolsky, S. A. \& Vetterling, W. T. 1989 Numerical Recipes (FORTRAN). Cambridge University Press.

Priede, J. \& Gerbeth, G. 1995 Hydrothermal wave instability of thermocapillary driven convection in a plane layer subjected to a uniform magnetic field. Adv. Space Res. 16 (7), 55.

Priede, J., Thess, A. \& Gerbeth, G. 1994 Thermocapillary instabilities in liquid metals - Hartmann number versus Prandtl number. Proc. Second Intl Conf. on Energy Transfer in MHD flows, Aussois (ed. A. Alemany, P. Marty \& J. P. Thibault), p. 571.

Rupp, R., Müller, G. \& Neumann, G. 1989 Three-dimensional time dependent modelling of the Marangoni convection in a zone melting configuration for GaAs. J. Cryst. Growth 97, 34.

SCHWARZ, H.R. 1993 Numerische Mathematik. Teubner.

Shen, Y., Neitzel, G. P., Jankowski, D. F. \& Mittelmann, H. D. 1990 Energy stability of thermocapillary convection in a model of the float-zone crystal-growth process. J. Fluid Mech. 217, 639 .

Velten, R., Schwabe, D. \& Scharmann, A. 1991 The periodic instability of thermocapillary convection in cylindrical liquid bridges. Phys. Fluids A 3, 267.

Wanschura, M., Kuhlmann, H. C. \& Rath, H. J. 1997 Linear stability of two-dimensional combined buoyant thermocapillary flow in cylindrical liquid bridges. Phys. Rev. E 55, 7036.

Wanschura, M., Shevtsova, V. M., Kuhlmann, H. C. \& Rath, H. J. 1995 Convective instability mechanisms in thermocapillary liquid bridges. Phys. Fluids 7, 912.

WiLson, S. K. 1994 The effect of a uniform magnetic field on the onset of oscillatory Marangoni convection in a layer of molten silicon. Micrograv. Sci. Technol. 7, 228.

Xu, J.-J. \& DAvis, S. H. 1984 Convective thermocapillary instabilities in liquid bridges. Phys. Fluids 27, 1102 . 